\title{
Lattice QCD, Spectral Functions and Transport Coefficients
}

\author{
Harvey B. Meyer* \\ PRISMA Cluster of Excellence, Institut für Kernphysik and Helmholtz Institut Mainz, \\ Johannes Gutenberg-Universität Mainz, D-55099 Mainz, Germany \\ E-mail: meyerh@uni-mainz.de
}

Lattice QCD simulations have provided key information on the equilibrium properties of strongly interacting matter at finite temperature. I review the progress recently achieved for nearequilibrium properties such as transport coefficients. Due to the use of the Matsubara formalism in lattice QCD, the latter properties are only accessible via a numerically ill-posed inverse problem. I discuss several methods to address the inverse problem more efficiently.

The 26th International Nuclear Physics Conference

11-16 September, 2016

Adelaide, Australia

${ }^{*}$ Speaker. 


\section{Introduction}

The subject of this talk is thermal strongly interacting matter in the temperature range $100 \mathrm{MeV} \lesssim$ $T \lesssim 1000 \mathrm{GeV}$. QCD matter in this temperature range is studied experimentally in high-energy heavy-ion collisions. At RHIC and the LHC, the temperature reached is sufficiently high for the produced QCD matter to be in the quark-gluon plasma (QGP) phase. The QGP was the dominant component of Standard Model matter in the early universe for the first few microseconds. While the cooling from the QGP to the low-temperature hadronic phase was a continuous crossover [1] and likely did not leave any detectable imprint, the production of weakly interacting particles in the early universe is sensitive to the detailed properties of the QGP. Two examples are axions, whose effective mass depends on the topological susceptibility of the QGP [2], and sterile neutrinos, whose production [3] depends on the vector and axial-vector spectral functions discussed below.

Studying QCD matter at finite temperature is part of the wider investigation of the QCD phase diagram as a function of temperature and baryon chemical potential $\mu_{B}$. Whether the thermal phase transition becomes stronger and reaches a critical point as $\mu_{B}$ increases is the object of continuing research. Here I describe the calculation of properties of QCD matter at $\mu_{B}=0$ using lattice QCD.

Lattice QCD provides a mathematically rigorous non-perturbative definition of QCD. It has also become a primary numerical computational tool. One of its applications is to provide the needed hadronic input for low-energy tests of the Standard Model. Another area where lattice QCD has had a tremendous impact is finite-temperature QCD, where various lattice collaborations have provided crucial input on static properties, such as the equation of state and quark number susceptibilities [4]. How to determine non-equilibrium quantities using lattice QCD is far less clear. The reason is that lattice QCD employs the Matsubara path-integral formalism, which is based on imaginary time $x_{0}$ with $0 \leq x_{0}<\beta$, where $\beta$ is the inverse temperature. Real-time aspects are therefore only indirectly accessible. Some of the questions we want to address are [5],

- What quasiparticles are there in the thermal system?

- How fast does an external perturbation dissipate in the system? For long wavelength perturbations, the rate is parametrized by transport coefficients (shear/bulk viscosity, diffusion coefficients, etc.). In linear-response approximation, the dissipation in real time of an externally induced, modulated electric charge density with wavevector $\vec{k}$ follows the generic diffusive behavior $e^{-D k^{2} t}$.

- What is the production rate of weakly coupled particles such as photons or dileptons? This type of question has applications both in heavy-ion collisions and in early-universe cosmology.

All three types of questions can be answered if certain spectral functions are known. To be concrete, consider the electromagnetic current $j^{\mu}=\frac{2}{3} \bar{u} \gamma^{\mu} u-\frac{1}{3} \bar{d} \gamma^{\mu} d-\frac{1}{3} \bar{s} \gamma^{\mu} s$ with $\gamma^{0 \dagger}=\gamma^{0}$ and $\gamma^{i}=-\gamma^{i \dagger}$. The lattice QCD approach to the spectral functions consists in computing Matsubara correlation functions

$$
G^{\mu v}\left(x_{0}, \vec{k}\right) \equiv \int d^{3} x e^{-i \vec{k} \cdot x}\left\langle j^{\mu}(x) j^{v}(0)\right\rangle
$$

and using the following dispersive relation ${ }^{1}$,

$$
G^{\mu v}\left(x_{0}, \vec{k}\right) \stackrel{\mu=v}{=} \int_{0}^{\infty} \frac{d \omega}{2 \pi} \rho^{\mu v}(\omega, \vec{k}) \frac{\cosh \left[\omega\left(\beta / 2-x_{0}\right)\right]}{\sinh [\omega \beta / 2]} .
$$

\footnotetext{
${ }^{1}$ It is also possible to formulate the dispersion relation in momentum space, $G\left(\omega_{n}, \vec{k}\right)=\int_{0}^{\infty} \frac{d \omega}{\pi} \omega \frac{\rho(\omega, \vec{k})}{\omega^{2}+\omega_{n}^{2}}$, however subtractions are needed to make the integral converge; it is e.g. sufficient to subtract the vacuum spectral function.
} 
For $\mu=v$, the spectral funtion $\rho^{\mu v}(\omega, \vec{k})$, whose temperature dependence is not indicated explicitly, is an odd function of $\omega$, and $\rho^{\mu v}(\omega, \vec{k}) / \omega$ is non-negative. In the vacuum $(T=0)$, the electromagnetic current can excite the $\rho, \omega, \phi$ mesons. Here the spectral function can be determined in a clean way in $e^{+} e^{-}$colliders. More generally, these vector excitations are expected to persist as quasiparticles in the hadronic phase, with somewhat modified masses, widths, etc. In addition, the low-frequency part of the spectral function encodes the transport properties of the thermal medium,

$$
\rho^{i i}(\omega, \overrightarrow{0}) \stackrel{\omega \rightarrow 0}{\sim} 6 \chi_{s} D \omega
$$

where $\chi_{s}=\int d^{4} x\left\langle j^{0}(x) j^{0}(0)\right\rangle$ is the static susceptibility of the electric charge and $D$ is the diffusion coefficient. This type of statement is called a Kubo formula. Finally, the differential production rate of photons per unit volume of the thermal medium is given by ${ }^{2}$

$$
d \Gamma_{\gamma}(\vec{k})=e^{2} \frac{d^{3} k}{(2 \pi)^{3} 2 k} \frac{\left[\rho^{i i}-\rho^{00}\right](k, \vec{k})}{e^{\beta k}-1}, \quad k \equiv|\vec{k}| .
$$

In the lattice QCD framework, Eq. (1.2) represents an inverse problem for the spectral function, given the correlator on the left-hand side. The state of the art is that the correlator is known to permille precision.

\subsection{Aspects of the inverse problem}

In the zero-temperature limit, $\beta \rightarrow \infty$ at fixed $x_{0}$, the inverse problem amounts to an inverse Laplace transform, a well-studied numerically ill-posed inverse problem. The study of low-lying hadrons in the vacuum is nonetheless highly successful, because their contribution dominates the correlator at late Euclidean times. At non-zero temperature, the restriction to $x_{0}<\beta$ makes matters worse. One diagnostic of the difficulty is provided by the following consideration. Due to the linearity of the inverse problem, one may take linear combinations of the Euclidean data points,

$$
\bar{\rho}(\bar{\omega}) \equiv \sum_{i=1}^{n} c_{i}(\bar{\omega}) G\left(x_{0}^{(i)}\right)=\int_{0}^{\infty} d \omega \rho(\omega) \underbrace{\sum_{i=1}^{n} c_{i}(\bar{\omega}) \frac{\cosh \left[\omega\left(\beta / 2-x_{0}^{(i)}\right)\right]}{2 \pi \sinh [\omega \beta / 2]}}_{\equiv \widehat{\delta}(\bar{\omega}, \omega)},
$$

where the coefficients $c_{i}(\bar{\omega})$ are chosen so that the 'resolution function' $\widehat{\delta}(\bar{\omega}, \omega)$ is as narrowly peaked around a given frequency $\bar{\omega}$ as possible and normalized, $\int_{0}^{\infty} d \omega \widehat{\delta}(\bar{\omega}, \omega)=1$. This is the idea behind the Backus-Gilbert method, used recently in $[6,7]$. The profile of the resolution function is illustrated in Fig. 1. The difficulty is that the resolution function only becomes narrower slowly with increasing number of data points $n$, and that this happens at the cost of large, sign-alternating coefficients $c_{i}$, whence the need for ultra-accurate lattice QCD data. This accuracy requirement means that sources of systematic errors, mainly discretization and finite-volume errors, must be brought down to a level comparable to the Monte-Carlo statistical errors. It is also worth noting that the positivity property of the spectral function is a valuable piece of information which is not exploited in the considerations above. The maximum entropy method and other Bayesian methods (see e.g. [8, 9]) do incorporate this aspect from the outset.

\footnotetext{
${ }^{2}$ For $\vec{k}$ along the z-direction, due to the Ward identity $\omega^{2} \rho^{00}=k^{2} \rho^{33}$, only $\rho^{11}+\rho^{22}$ contributes in Eq. (1.4); however the combination $\rho^{i i}-\rho^{00}$ is the one needed for the dilepton production rate as well.
} 


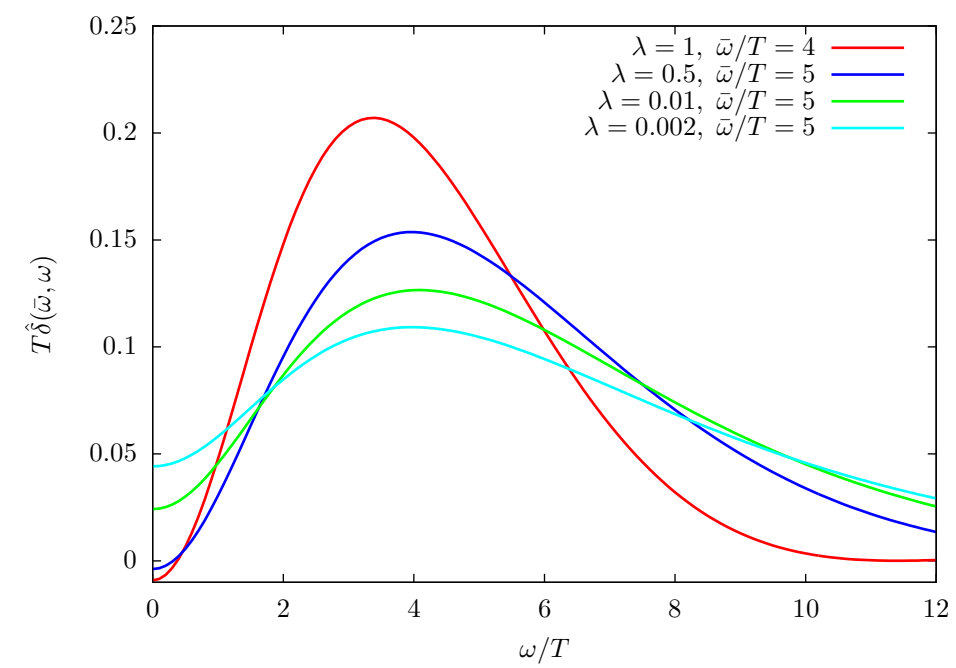

Figure 1: Resolution functions $\widehat{\delta}(\bar{\omega}, \omega)$ at $\bar{\omega}=4 T$ and $5 T$ for the inverse problem (1.2) on a lattice with $\beta / a=24$ using data points $x_{0} / a=5,6, \ldots, 12$; from [6]. Without prior knowledge, only the 'fudged' version $\bar{\rho}(\bar{\omega})=\int_{0}^{\infty} d \omega \rho(\omega) \widehat{\delta}(\bar{\omega}, \omega)$ of the spectral function can be determined. The optimal, narrowest resolution function $(\lambda=1)$ requires large, sign-alternating coefficients $c_{i}$, leading to large stastistical errors on $\bar{\rho}(\bar{\omega})$. In practice, as a compromise, the resolution function is chosen less narrow $(\lambda<1)$ to stabilize the result.

\section{Recent progress in vector and axial-vector channels}

Beyond the vector channel, which gives access to the diffusion constant, the shear and bulk viscosity are of great interest, given the prominent role of these transport coefficients in the description of heavy-ion collisions. The viscosities are extracted via Kubo formulae from the spectral functions associated with the energy momentum tensor. Furthermore, the properties of quarkonia and heavy-light mesons serve as hard probes of the created medium in heavy-ion phenomenology. In the hadronic phase, the properties of the pion quasiparticle are of particular interest, it being the single most abundant hadron in the medium. In the following, we review several channels where progress has recently been made.

\subsection{The vector correlator and the diffusion coefficient}

Figure 2 shows a recent calculation [7] of the isovector vector current correlator on lattices of size $N_{t} \times 64^{3}$ at vanishing spatial momentum, the temperature being varied between 0.8 and $1.7 T_{c}$ at fixed lattice spacing by choosing $N_{t}=12,16,20,24$ and 128 (the latter corresponding to the vacuum). Here various explicit fit ansätze for the spectral function were applied. The leading large-frequency behavior $\rho^{i i}(\omega) \propto \omega^{2}\left(1+\alpha_{s} / \pi+\ldots\right)$ is temperature-independent, and therefore all temperatures were fitted simultaneously. In addition, an exact sum rule, $\int_{0}^{\infty} \frac{d \omega}{\omega}\left[\rho^{i i}(\omega, T)-\right.$ $\left.\rho^{i i}\left(\omega, T^{\prime}\right)\right]=0$ was exploited to further constrain the spectral functions.

While the calculation is performed at relatively small lattice spacings, an extrapolation to the continuum is still missing. In the 'quenched' approximation, where the effects of the quarks on the QGP are neglected, one collaboration has been able to take the continuum limit [10]. Recent results for the diffusion coefficient $D$ of light-quarks are displayed in Fig. 3. In the QGP, lattice calculations yield very low values, $D \approx 1 /(\pi T)$, to be compared with the leading perturbative 

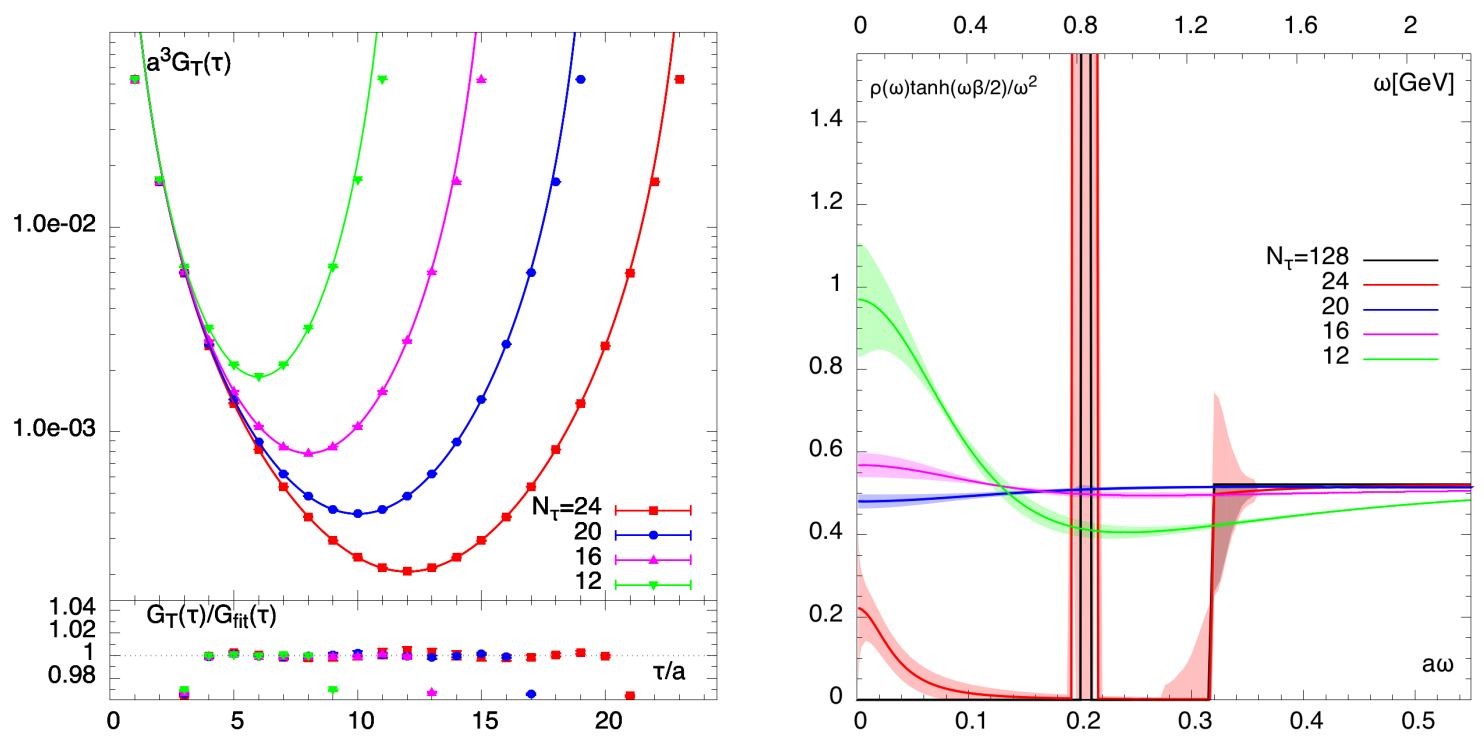

Figure 2: Left: lattice vector isovector correlators as a function of imaginary time at four different temperatures between 0.8 and $1.7 T_{c}$, where $T_{c} \simeq 203 \mathrm{MeV}$ at the simulation parameters; the statistical precision on the correlators is about two permille or better. Right: the result of fits to the lattice data for the spectral functions. By the Kubo formula (1.3), the ordinate at $\omega=0$ corresponds to $3 \chi_{s} D / T$. Fig. from [7].

prediction [14] $D \approx 1.7 / T$ for a reasonable value of the strong coupling constant $\alpha_{s}$; in strongly coupled $\mathscr{N}=4$ super-Yang-Mills theory [15], the result is $D=1 /(2 \pi T)$. However, one should bear in mind that all lattice results in the left panel of Fig. 3 implicitly assume that no narrow transport peak is present around $\omega=0$. In other words, if a long relaxation time-scale (compared to the thermal time scale $1 / T$ ) were present, as is certainly the case at very high temperatures, it would be missed.

In addition to the diffusion of light quarks, one may consider the diffusion in the opposite limit of a very massive (static) quark (see the data point [11] labelled 'heavy-quark' in Fig. 3). The large mass scale present allows one to simplify the problem somewhat: the heavy-quark diffusion coefficient can be related by an Einstein relation to a momentum diffusion coefficient, which is determined by a gluonic force-force correlator [12]. The spectral function describing the latter is smooth even at weak coupling, therefore the calculation is expected to be better controlled.

\subsection{The photon production rate}

As described in the introduction, the vector spectral function on the light-cone $(\omega=|\vec{k}|)$ gives access to the differential photon rate. The authors of [16] define an effective diffusion coefficient,

$$
D_{\text {eff }}(k)=\left[\rho^{i i}(k, \vec{k})-\rho^{00}(k, \vec{k})\right] /\left(4 \chi_{s} k\right)
$$

which coincides with $D$ in the limit $k \rightarrow 0$. As illustrated by the right panel of Fig. 3, while the lattice calculation of $D_{\text {eff }}(k)$ is reasonably well controlled for $k \approx 2 \pi T$, the uncertainty increases rapidly as $k$ is decreased. The reason is that one expects a more slowly varying spectral function at non-zero spatial momentum. In the study [16], a polynomial fit ansatz was used up to $\omega=$ $\sqrt{k^{2}+\pi^{2} T^{2}}$, and advanced perturbation theory predictions were exploited at larger frequencies. 

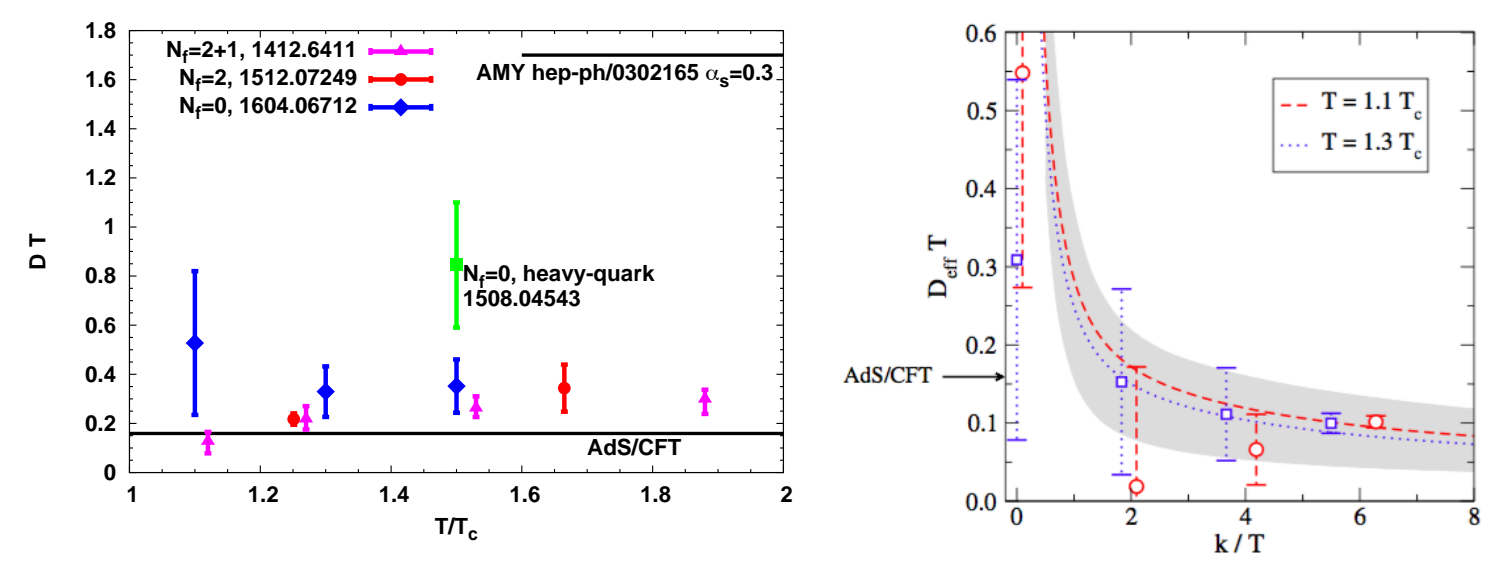

Figure 3: Left: Selected recent results for the diffusion coefficient $D$ of light-quarks $[13,7,10]$, compared to the weak-coupling prediction [14] and AdS/CFT prediction [15]. In addition, the data point explicitly labelled "heavy-quark" is obtained in the static limit [11]. Right: The effective, momentum-dependent diffusion coefficient that determines the photon production rate; see Eq. (2.1). Fig. from [16].

\subsection{The pion quasiparticle in the hadronic phase}

Chiral symmetry is spontaneously broken for $T<T_{c}$, and the chiral condensate $\langle\bar{\psi} \psi\rangle$ is nonvanishing. Goldstone's theorem then implies a divergent spatial correlation length $m_{\pi}^{-1}$ in the limit $m_{u, d} \rightarrow 0$. But also, a massless real-time excitation exists: the pion quasiparticle. Its dispersion relation generically takes the form

$$
\omega_{\vec{p}}=u(T)\left(m_{\pi}^{2}(T)+\vec{p}^{2}\right)^{1 / 2}+\ldots
$$

at low momenta [17]. For $T \lesssim 100 \mathrm{MeV}$, the two-loop chiral perturbation theory prediction for the pion quasiparticle mass $\omega_{\overrightarrow{0}}$ indicates that the quasiparticle mass is reduced [18]. However, the effect of the other hadrons rapidly become important above $T \simeq 100 \mathrm{MeV}$; therefore a lattice QCD calculation is needed to quantify the temperature dependence of the pion quasiparticle mass.

A key point is that the pion parametrically dominates the Euclidean two-point function of the axial charge density and of the pseudoscalar density at $x_{0}=\beta / 2 \approx 0.6 \mathrm{fm}$ and $|\vec{p}| \lesssim 300 \mathrm{MeV}[19,6]$. Treating the pion in the narrow-width approximation and using the ansatz

$$
\rho^{A}(\omega, \vec{p}) /(2 \pi)=a_{1}(\vec{p}) \delta\left(\omega^{2}-\omega_{\vec{p}}^{2}\right)+a_{2}(\vec{p})\left(1-e^{-\omega \beta}\right) \theta(\omega-c)
$$

for the spectral function of the axial charge density, a good description of the Euclidean correlator is obtained. The pion thermal width is parametrically suppressed [17]; a quantitative estimate based on the virial expansion [20] yields a value $\gamma_{\pi}^{T} \approx 20 \mathrm{MeV}$ at $T \approx 100 \mathrm{MeV}$. The pion residue has the form [17, 6] $a_{1}(\vec{p})=f_{\pi}^{2}(T)\left(m_{\pi}^{2}(T)+\vec{p}^{2}\right)$. The screening pion mass $m_{\pi}(T)$ and decay constant $f_{\pi}(T)$ can be determined reliably from static screening correlation functions. For a zerotemperature pion mass $m_{\pi}=(267 \pm 2) \mathrm{MeV}$ and $T=170 \mathrm{MeV}$, we obtain $u=0.75(2)$ and $\omega_{0}=$ $(223 \pm 4) \mathrm{MeV}$ for the quasiparticle mass. The change in the quasiparticle mass is thus sizeable.

\section{Conclusion}

There has been significant progress in the lattice study of real-time properties of thermal QCD 

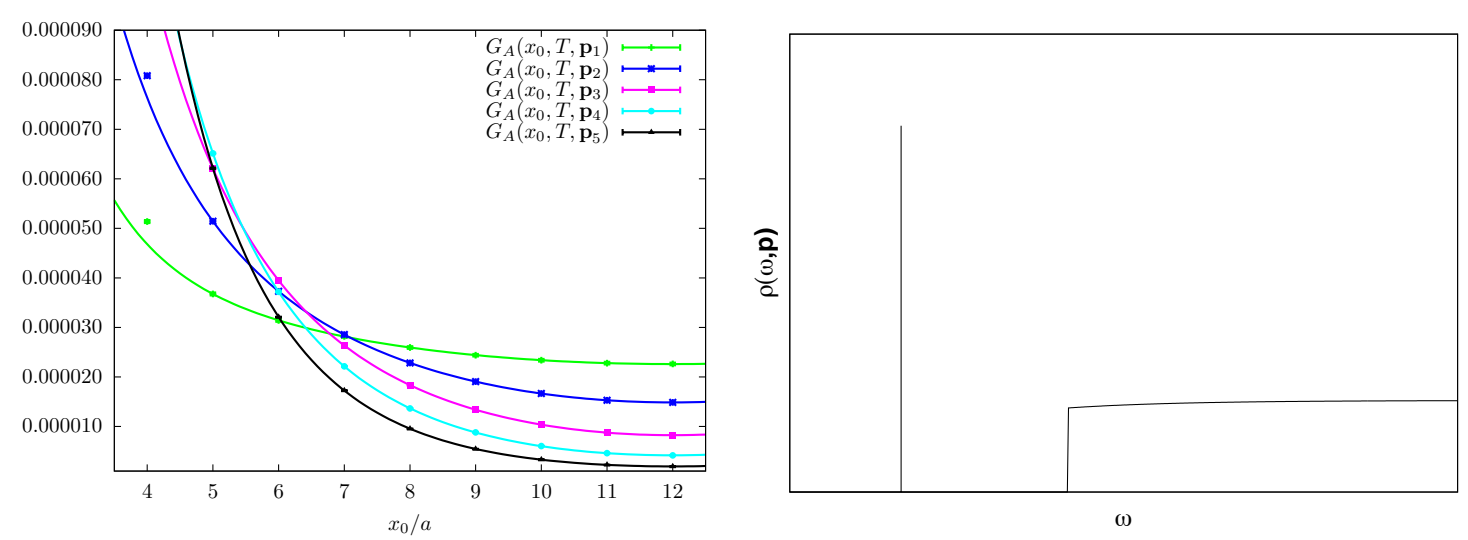

Figure 4: The Euclidean correlator of the axial charge density at various spatial momenta (left; from [6]), and (right) its description via a spectral function containing the pion pole and a continuum (see Eq. (2.3)).

matter via spectral functions, in spite of the tremendous challenge posed by the inverse problem inherent in the methodology. Lattice data on rather fine lattices have become available, the continuum limit has been taken in the quenched approximation and the statistical precision in several channels is now at the permille level. The determination of the shear viscosity proceeds similarly to the calculation of the quark diffusion constant, but since it involves the (flavor-singlet) energy-momentum tensor, it is numerically much more demanding. Nonetheless, since the exploratory calculations [21, 22], new calculations have appeared recently in the pure $\mathrm{SU}(3)$ gauge theory [23, 24].

One open issue to my mind is the fact that the hadron resonance gas model, which assumes that the hadrons undergo no thermal modification, describes static observables such as the equation of state and quark number fluctuations rather well (see e.g. [25]), while the dedicated study of the pion quasiparticle described above does indicate a sizeable reduction in its mass.

I wish to thank the organizers for the invitation and for running this major conference very smoothly. I also warmly thank my collaborators (Ref. [6]). My own research is supported by the DFG grant ME 3622/2-2 QCD at non-zero temperature with Wilson fermions on fine lattices.

\section{References}

[1] Y. Aoki, G. Endrodi, Z. Fodor, S. Katz, and K. Szabo, The Order of the quantum chromodynamics transition predicted by the standard model of particle physics, Nature 443 (2006) 675-678, [hep-lat/0611014].

[2] S. Borsanyi et. al., Calculation of the axion mass based on high-temperature lattice quantum chromodynamics, Nature 539 (2016), no. 7627 69-71, [arXiv: 1606.0749 ].

[3] T. Asaka, M. Laine, and M. Shaposhnikov, On the hadronic contribution to sterile neutrino production, JHEP 06 (2006) 053, [hep-ph/ 060520 9].

[4] H.-T. Ding, F. Karsch, and S. Mukherjee, Thermodynamics of strong-interaction matter from Lattice QCD, Int. J. Mod. Phys. E24 (2015), no. 10 1530007, [arXiv: 1504.0527 ].

[5] H. B. Meyer, Transport Properties of the Quark-Gluon Plasma: A Lattice QCD Perspective, Eur.Phys.J. A47 (2011) 86, [arXiv:1104.3708]. 
[6] B. B. Brandt, A. Francis, H. B. Meyer, and D. Robaina, Pion quasiparticle in the low-temperature phase of QCD, Phys. Rev. D92 (2015), no. 9 094510, [arXiv: 1506.0573 ].

[7] B. B. Brandt, A. Francis, B. Jaeger, and H. B. Meyer, Charge transport and vector meson dissociation across the thermal phase transition in lattice QCD with two light quark flavors, Phys. Rev. D93 (2016), no. 5 054510, [arXiv:1512.0724].

[8] M. Asakawa, T. Hatsuda, and Y. Nakahara, Maximum entropy analysis of the spectral functions in lattice QCD, Prog. Part. Nucl. Phys. 46 (2001) 459-508, [hep-lat/ 0011040 ].

[9] Y. Burnier and A. Rothkopf, Bayesian Approach to Spectral Function Reconstruction for Euclidean Quantum Field Theories, Phys.Rev.Lett. 111 (2013) 182003, [arXiv:1307.6106].

[10] H.-T. Ding, O. Kaczmarek, and F. Meyer, Thermal dilepton rates and electrical conductivity of the QGP from the lattice, Phys. Rev. D94 (2016), no. 3 034504, [arXiv: 1604.0671 ].

[11] A. Francis, O. Kaczmarek, M. Laine, T. Neuhaus, and H. Ohno, Nonperturbative estimate of the heavy quark momentum diffusion coefficient, Phys. Rev. D92 (2015), no. 11 116003, [arXiv:1508.0454].

[12] S. Caron-Huot, M. Laine, and G. D. Moore, A way to estimate the heavy quark thermalization rate from the lattice, JHEP 04 (2009) 053, [arXiv: 0901.1195$].$

[13] G. Aarts, C. Allton, A. Amato, P. Giudice, S. Hands, and J.-I. Skullerud, Electrical conductivity and charge diffusion in thermal QCD from the lattice, JHEP 02 (2015) 186, [arXiv: 1412 . 6411].

[14] P. Arnold, G. D. Moore, and L. G. Yaffe, Transport coefficients in high temperature gauge theories. II: Beyond leading log, JHEP 05 (2003) 051, [hep-ph/ 0302165 ].

[15] G. Policastro, D. T. Son, and A. O. Starinets, From AdS / CFT correspondence to hydrodynamics, JHEP 0209 (2002) 043, [hep-th/ 0205052 ].

[16] J. Ghiglieri, O. Kaczmarek, M. Laine, and F. Meyer, Lattice constraints on the thermal photon rate, Phys. Rev. D94 (2016), no. 1 016005, [arXiv: 1604 .0754].

[17] D. T. Son and M. A. Stephanov, Real-time pion propagation in finite-temperature QCD, Phys. Rev. D66 (2002) 076011, [hep-ph / 0204226 ].

[18] D. Toublan, Pion dynamics at finite temperature, Phys.Rev. D56 (1997) 5629-5645, [hep-ph/9706273].

[19] B. B. Brandt, A. Francis, H. B. Meyer, and D. Robaina, Chiral dynamics in the low-temperature phase of QCD, Phys.Rev. D90 (2014) 054509, [arXiv: 1406.5602 ].

[20] H. Leutwyler and A. V. Smilga, Nucleons at finite temperature, Nucl. Phys. B342 (1990) 302-316.

[21] H. B. Meyer, A calculation of the shear viscosity in SU(3) gluodynamics, Phys. Rev. D76 (2007) 101701, [arXiv:0704.1801].

[22] H. B. Meyer, Transport properties of the quark-gluon plasma from lattice QCD, Nucl. Phys. A830 (2009) 641c-648c, [arXiv:0907.4095].

[23] S. W. Mages, S. Borsanyi, Z. Fodor, A. Schaefer, and K. Szabo, Shear Viscosity from Lattice QCD, PoS LATTICE2014 (2015) 232.

[24] N. Astrakhantsev, V. Braguta, and A. Kotov, Temperature dependence of shear viscosity of SU(3)-gluodynamics within lattice simulation, arXiv:1701.0226.

[25] A. Bazavov et. al., Additional Strange Hadrons from QCD Thermodynamics and Strangeness Freezeout in Heavy Ion Collisions, Phys. Rev. Lett. 113 (2014), no. 7072001 , [arXiv:1404.6511]. 